\title{
Circular digital devices: lessons about the social and planetary boundaries
}

\author{
Mireia Roura \\ Universitat Politècnica de Catalunya \\ Barcelona, Spain \\ m.roura@upc.edu \\ Leandro Navarro \\ Universitat Politècnica de Catalunya \\ Barcelona, Spain \\ leandro.navarro@upc.edu
}

\author{
David Franquesa \\ Universitat Politècnica de Catalunya \\ Barcelona, Spain \\ david.franquesa@upc.edu \\ Roc Meseguer \\ Universitat Politècnica de Catalunya \\ Barcelona, Spain \\ meseguer@ac.upc.edu
}

\begin{abstract}
The digital world can be part of the solution to the coexistence of people and the planet, with large efficiencies gained through digital solutions, but it is part of the problem too, as it requires vast natural resources for digital devices in terms of participation. The challenge lies in satisfying the demand for digital devices for everyone, particularly for those with scarce resources, while preserving planetary limits. We have implemented and optimised a circular economy ecosystem that -through donation, refurbishment and traceability- delivers locally reused digital devices at a fair economic and environmental cost to citizens in need, providing satisfactory computing services and reducing the demand to manufacture new devices, which instead must be more durable. Following the sourcing of 10,000 computers, 1,000 have been successfully refurbished by local social enterprises and used by citizens in the city of Barcelona as a common good, herein we present an analysis of the achievements, limitations, and conditions of this model, which has already been replicated in other areas. It is feasible, and we also explore its scalability and sustainability in economic, social, and environmental contexts. We identify critical success factors, such as the roles of local public or private donors, social-support organisations, and ecosystem facilitators, in mediating among all actors involved.
\end{abstract}

\section{KEYWORDS}

Circular economy, digital devices, licences, use-value, algorithms, platform cooperativism

\section{Reference Format:}

Mireia Roura, David Franquesa, Leandro Navarro, and Roc Meseguer. 2021. Circular digital devices: lessons about the social and planetary boundaries. In LIMITS '21: Workshop on Computing within Limits, fune 14-15, 2021.

Permission to make digital or hard copies of part or all of this work for personal or classroom use is granted without fee provided that copies are not made or distributed for profit or commercial advantage and that copies bear this notice and the full citation on the first page. Copyrights for third-party components of this work must be honored For all other uses, contact the owner/author(s).

LIMITS '21, fune 14-15, 2021,

(C) 2021 Copyright held by the owner/author(s).

\section{INTRODUCTION}

Participation in the digital world requires digital devices to access the Internet, and that makes it part of our planetary problem. However, if the digital world can serve to satisfy the needs of all humans, especially those penalised by limited resources, while staying within planetary limits, it could be part of the solution too. Access to digital devices for all is about overcoming problems with availability and the economic barrier of affordable prices for otherwise-excluded citizens, reconciling planetary limits with human needs based on a dignified, just, and sustainable lifestyle [45].

The situation of vulnerable populations and the COVID-19 pandemic has raised awareness of an acute situation. Digital inequality is widening an education divide during confinement and homeschooling, so governments have had to improvise solutions, given their role as guarantors of universal education (SDG 4). There are different ways to supply devices for those who lack one: the mass purchase of newly manufactured devices or the reuse of alreadybuilt devices ${ }^{1}$. While the first linear economy strategy has proved not to be resilient in face of the demand peaks that have resulted in disrupted stocks and long delays in distribution, the second strategy is part of the circular economy, where devices are donated, collected locally, and refurbished for maximal lifespan, ensuring final recycling (environmental limit) and contributing to the social inclusion of those who use these devices: affordable devices as a commons under shared property [42]. Balancing social and environmental needs and using creative experimentation in communities are key concerns in our research.

Our method combined participatory action research [48], more specifically network action research [17], with iterative experimentally driven research [25]. After several years' work and beyond 10,000 computers being processed, mainly by 15 social organisations, such projects have been initiated and replicated in several cities [23] We focus our results on 1,079 devices managed as a commons in Barcelona, refurbished by six reuse centres. The main contribution of this paper is an analysis of our experience of a circular economy of digital devices, from the theory and initial knowledge showing feasibility to the experience and evidence acquired from the expansion, consolidation, and replication of the model, including the lessons learned from the COVID-19 pandemic. We discuss

\footnotetext{
${ }^{1}$ Corporate social responsibility of companies, volunteer-based initiatives to donate personal devices, public donors such as in Barcelona, Amsterdam, combined with NGO and social enterprises such as ComputerAid or eReuse [24]
} 
the alignment of social, environmental, and economic impacts, as refurbishing a device may not be economically profitable or replace demand for new devices, even if it has reusability potential. We quantitatively analysed the characteristics of these 1,079 traced devices (hours of use during their first life, processors, and type of donor) and followed them during the reuse phase through interviews with final beneficiaries. We perform a cost-benefit analysis of cases in Barcelona [28]. Finally, we discuss feedback from the reuse centres and other stakeholders involved.

The paper is organised as follows. In section 2, we introduce the role of the circular economy for digital devices in the context of sustainability and social and environmental limits to address. Section 3 describes the eReuse model in its current form. Section 4 presents the results achieved by eReuse in the recent years of expansion, consolidation, and replication. We discuss the results and lessons learnt in section 5. Related work is discussed in section 6, with concluding remarks and future work in section 7 .

\section{THE CIRCULAR ECONOMY OF DIGITAL DEVICES}

Sustainable development is about meeting "the needs of the present without compromising the ability of future generations to meet their own needs" (the Brundtland Commission Report, 1987 [37]), supporting economic development while simultaneously sustaining the ability of natural systems to provide the natural resources and ecosystem services on which the economy and society depend. [49]. However, development should not be confused with growth: growth relates to environmental degradation or "tragedy of the commons" [29], while equilibrium relates to sustainability as reported in the Club of Rome report Limits to Growth in 1972 [35] and the UN Our Common Future report in 1987 [37]. The sustainability of human development and progress is dependent on reconnection to the capacity of the biosphere and essential ecosystem services [46]. Sustainable development can be thought of in terms of three pillars: economic, environmental, and social.

Circularity-"designing out waste and pollution, keeping products and materials in use, and regenerating natural systems" [19]-is a key way to implement sustainable development. In more detail, it is usually defined in terms of the three Rs: reducing materials and waste, reusing products, and recycling materials, enabling products and services to be in closed loops or cycles ideally indefinitely. An outcome-based definition that has been suggested is "the circular economy is a new economic model for addressing human needs and fairly distributing resources without undermining the functioning of the biosphere or crossing any planetary boundaries" [36].

The Intergovernmental Panel on Climate Change (IPCC) 2018 special report [34] has defined targets to address the impacts of a $1.5^{\circ} \mathrm{C}$ warming of the planet. To meet this goal, the IPCC said we must cut emissions to net zero by 2050 . If we focus on digital devices and the information and communication technology (ICT) world, currently more than 6 billion new ICT goods are sold annually worldwide. There are estimates of 1.5 billion smartphones [47]. It is thus imperative that we decarbonise our planet to tackle the environmental crisis. According to the International Telecommunication Union (ITU), ITU-T [31] we need to reduce the environmental footprint of the ICT industry to half the footprint of 2015 by 2030 .
Fellner et al [15] studied scenarios of only recycling without reuse, and estimated that our $\mathrm{CO} 2$ footprint would be reduced by just $1.6 \%$. In addition to this clearly inadequate figure, in 2019 and based on the current documentation collection, recycling only recovered US $\$ 10$ billion of the value of the raw materials contained in the e-waste generated $-\$ 57$ billion globally [16]. This figure is, among other things, higher than the 2020 GDP of Lithuania.

Among the raw materials from e-waste, iron, copper, and gold are the most valuable. Serious social and environmental impacts have been reported in the production and extraction of gold [18] and other rare materials, like the tantalum necessary to obtain coltan for mobiles. Violations of workers' rights along the manufacturing chain are also well known.

In the context of digital devices, circularity aims at achieving the best use of these devices by extending their lifespan, which helps in decarbonising the environment. Moreover, circularity can also help reduce social inequality by delivering computing devices to and creating jobs for a large portion of the population that are not among those who have sufficient disposable income to buy the trendiest digital devices and brands in the global market. Even if we can afford these, they are still detrimental to our natural environment, society, and economy.

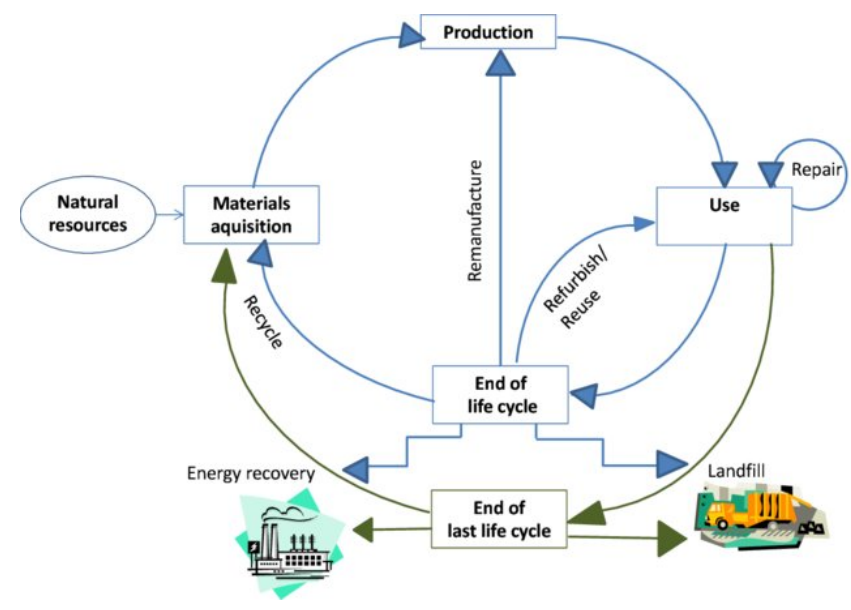

Figure 1: The flows in the life cycle of ICT goods.

The representation in Figure 1 [30] makes an important distinction for ICT goods between the end of a usage cycle, which can end with recycling, landfill, or return to use through refurbishment/reuse, and the end of the final life cycle, which goes again to recycling or a landfill.

\section{THE eREUSE MODEL}

Digital devices (computers, tablets, mobiles) that are no longer used are a resource that can be utilised for local social inclusion and participation. Our vision is that public and private organisations act in the common good for a better, more inclusive, and environmentally friendly Internet by donating their disposed devices to such social enterprises as reuse centres, which repair, refurbish, and then distribute them to citizens that need devices to participate via the Internet in education and socioeconomic activities in their communities. This secondhand market creates social inclusion through 
jobs and plenty of devices at a fair price. It also feeds a circular economy that improves local socioeconomic and environmental conditions.

The challenge lies in the collection and refurbishment of computers and mobiles for reuse and maximal lifespan, ensuring final recycling. Digital devices that end a first-usage phase from individuals and public/private organisations can have a second life, instead of being dumped or shredded. Refurbishment and repair, carried out either individually or more commonly by social enterprises, can prepare these devices for new use (reuse) and offer them to final users as beneficiaries at cost, which creates an inclusive offer for cheap but useful devices.

For that, there is a need for collaborative bootstrapping by local circular economy ecosystems across all stakeholders in the ICT reverse-supply chain of reuse and recycling. eReuse has developed fora to coordinate different stakeholders in locations that can exchange complementary resources and skills, balance supply and demand, share costs, and help one another, with device donors, refurbishment technicians, citizen-support organisations, and recyclers working together as part of a pooled-resource system of secondhand digital devices in extended use [20]. We call these "local circuits". The beneficiaries of our activities are those in municipal social-support programs, interested in secondhand computers, and schools, public facilities, and families supported by neighbourhood social-support organisations.

For circular use, devices really need to provide a substitute to the demand for newly manufactured devices, and tracing, certifying, and measuring circularity (chain of custody) of products, members, and platforms needs to be in place to ensure we can achieve and measure the best social, economic, and environmental impacts. This coordination and community development requires the establishment of common local processes to support the reuse of electronics. To ensure efficiency, transparency, and accountability in the reversesupply chain, we developed open-source software ${ }^{2}$ [24] to extract details quickly and easily for diagnosis and automation of inventory, triage and preparation of secondhand devices, and generation of QR codes to stick on devices for traceability, as well as data formats and tools to collect and store data on the lifespan of each device, and finally for computing open data on impacts and for reports. This functionality is in line with the so-called "digital product passport", a concept that is gaining attention in the political agenda and expected to provide consistent digital information on the origin, composition, repair, and dismantling options for a product, as well as on its handling at the end of its service life [1].

eReuse started in 2013, reaching an important milestone in 2015 with the launch of a computer-donation campaign. More than 10,000 computers have been processed with the eReuse software tools. We have about 15 active social organisations, and we have created local circuits in Barcelona and Madrid.

In a typical circuit, a donor organisation (public or private) donates decommissioned devices that are collected by a social enterprise that brings these computers in pallets to a refurbishment facility operated by a social enterprise or a reuse centre. There, the devices are put in a rack and inspected and the data wiped, tested, and reinstalled with (usually) a Linux operating system, in parallel

\footnotetext{
${ }^{2}$ https://www.eReuse.org/software/https://github.com/eReuse
}

with eReuse-software tools. Those that do not pass the test are put in a cage for recycling and recorded in our system as prepared for recycling. Those that pass the test are cleaned, checked in more detail, sometimes upgraded (battery, RAM, storage), labelled, and stored for sale, with the cost sponsored by a third party, although it is recommended that the final beneficiary contributes something as a commitment. Usually, social support or public organisations, and sometimes individuals, acquire these devices, which are brought to end users with the commitment to return the devices to the intermediary organisation after usage for another refurbishment or final recycling. The eReuse software records all these transfers and can generate a complete provenance log for each device about its lifespan, without revealing any personal details about the users.

We have developed agreements with public and private device donors, social organisations (NGOs) working with end users, and social enterprises involved in social-inclusion programs working with refurbishment and recycling. These agreements allow us to obtain data about devices (chain of custody), aggregate data, and analyse social (usefulness of computing hours enabled) and environmental impact (CO2 savings).

We have developed a service-focused ("servitised") business model where users pay for computing as a service, for instance the number of operational computers in a school classroom, and actors in the circuit ensure performance maintenance, upgrades, and replacements to ensure the service contract for a given period in exchange for a monthly or yearly fee. This ensures they have the computing they need, but ownership remains in the circuit, which ensures the units perform well until they cannot satisfy anyone and are finally recycled and some parts reused. Recyclers are specialised in e-waste, and can be public, commercial, or social enterprises dedicated to this process. They can contribute to eReuse data by recording the devices they receive through scanning any $\mathrm{QR}$ codes on those computers.

eReuse delivers training of actors on different aspects of device refurbishment, as well as dissemination activities to raise awareness about environmental issues in ICT. Overall, this generates datasets on impacts on the durability of devices we process [22]. All combined results in key activities and actors that improve the longevity of devices through potential new reuse cycles for helping more people with devices mitigate the impact of ICT on the planet.

\section{RESULTS}

We have produced an open dataset [22] on devices and parts, with over 10,000 computers included by the end of 2020 . These correspond to diverse initiatives. We have limited the study to only 15 resellers with operations in Spain that have accepted the eReuse device-data commons license [21]. We focus our analysis on cases in the Barcelona metropolitan area and Madrid, where we have been more involved and studied in detail.

In Barcelona, we have 1,079 devices in shared property as a device commons, with more under individual property regimes. The devices have been donated by 45 donors and received by 84 entities, such as schools, public facilities, NGOs, and final beneficiaries. Refurbished devices are prepared by workers of six social enterprises or reuse centres associated with the Barcelona circuit. Devices have 
a refurbishment cost that is paid by sponsors or recipients to refurbishment technicians (as processing cost, since devices are donated) of $€ 40-140$ each.

In 2018, the eReuse circuit model and the use of its software was replicated in Madrid by the Asociación Cultural La Kalle, a nonprofit association in the neighbourhood of Vallecas that (among others) seeks the social and labour inclusion of young, vulnerable people through promotion of the reuse of electronic devices. It promoted an ecosystem with another two entities (Vortex and RecuMadrid), called the Sempiterna circuit. Since it started, it has reused 950 devices donated by 14 companies and given to 23 intermediary entities (NGOs and schools).

The main overall impact and outcomes are:

- Reduction in environmental impact of ICT, with CO2-equivalent impact estimates.

- Universal access to computing devices through the opportunity to use these low-cost secondhand computers, measuring additional computing hours provided.

- Creation of jobs in computer refurbishment, linked to device collection and refurbishment.

- Reduction in ICT e-waste and environmental impact of ICT, with more durable devices through reuse.

- Development of software for more efficient (time, quality) processing of ICT devices, which implies less refurbishment time per device, resulting in lower processing costs and higher efficiency and salaries for refurbishment technicians.

- Collection of reliable open-source data to promote circularity (chain of custody), promote good behaviour, and quantify and certify impacts, for instance, with durability statistics about different models and brands that can assist purchase decisions and requesting more durable designs.

The following section presents and discusses key lessons learnt, supported by detailed results from the shared experience of most eReuse participants.

\section{DISCUSSION AND LESSONS LEARNT}

Through the analysis of several cases with running business models as part of the eReuse model, we highlight four crucial challenges that need to be considered for this or other models to be successful We have identified key elements for each challenge, crucial to ensure the system succeeds or fails in terms of economic sustainability (income and contribution), balance (across devices' supply and demand), ensure suitability and quality at affordable price to meet final users' needs and appropriation, and ability to scale to the target demand. We discuss the lessons learned, supporting details about results from our experience, and any deviations from initial expectations.

\subsection{Who bears the cost of putting reused devices into circulation?}

The circular economy must pay the bills. This requires someone willing to donate or in need of a reused device and someone/an entity willing to pay the necessary costs to deliver it. The limitation of our model is that in most cases, the population in need is unable to pay. In fact, the cost of putting devices into circulation is covered in some cases by sponsors, corporate social responsibility initiatives, or public services, among others. Nevertheless, having a business model with only this target audience is hardly sustainable or viable in the long term.

One way is to expand the market, breaking the paradigm of reuse being inevitably linked to poverty. This should be done without losing the principles of eReuse, not forgetting that there are many final users that are not able to pay. Ways to achieve this rely on pricing devices according to use value: for the same refurbishment cost of a donated device, users may be willing to pay more for a device with better performance. That economic margin over the refurbishment cost can compensate for the cost for other devices. This formula raises an ethical problem not yet resolved, since the consequence is that those who can pay more have access to better computers than those who can pay less.

Supporting results:

Revenue from the activity associated with direct computer sales comes to $€ 15,000-18,000 /$ year (donalo.org) or $€ 20,000 /$ year (Asociación Cultural La Kalle). Both figures show that short-term and long-term economic sustainability are dependent upon subsidies or financial support from private entities, unless structured responsible public procurement or tenders are involved.

Accountability and impacts. To collect details on devices across their lifespan brings accountability, allows the determination different impacts, and shows the positive externalities of reuse (such as environmental savings, social inclusion, and local jobs created). Moreover, tracing computers with eReuse software makes the reversesupply chain transparent to all stakeholders in the ecosystem. These actions can attract additional economic contributions from public or private organisations to help projects be more sustainable. Supporting results:

The Barcelona City Council has a computer park of around 12,000 computers that are used quite efficiently, taking into account that their current internal life is $8-9$ years, despite accounting depreciation being 4 years. Accounting depreciation has been pointed to as a barrier towards the adoption of circular revenue models, because depreciation of products more quickly than the expected lifespan due to tax and accounting objectives creates a perception of devices without any value [9].

In November 2016, with the aim of promoting the circular economy, Barcelona Activa signed an agreement with eReuse for a pilot. This was an example of value creation for other public/private sectors to follow. After the Barcelona Activa agreement, many donations from the public sector were received: Barcelona Regional, Barcelona Ecología Urbana, Catalonia Waste Agency, and Barcelona Libraries (Diputació de Barcelona). The example set by these public bodies was followed by 37 private organisations. All of them were motivated by three clauses that the Interreg [14] and NGI [38] policy initiatives support: using templates for transfer of ownership or possession of devices with different clauses to regulate circular and social impacts specifying end-user target segments, identifying target refurbishing organisations with proven impact accounting and redistributing devices transparently and efficiently, and getting impact-data and circularity reports on the traceability of devices over multiple owners and possessors without affecting privacy and ensuring data integrity in order to provide feedback to donors in terms of retribution and certificates on the circular impact of the transition (such as $\mathrm{CO} 2$ avoided, hours of extended usage, segment 
of final-user beneficiaries, and amount of hazardous waste recycled and recovered).

Despite this, we are aware that to produce fully circular devices, Barcelona City Council would need to start by procuring devices designed for a durable, repairable, and reusable lifespan, including second life. We believe these considerations to be a logical and necessary step, after the city council's commitment in 2016 to Electronic Watch, an entity that helps public-sector organisations to work together, and to collaborate with civil-society monitors in production regions to protect the rights of workers in their electronics supply chain.

Dumping, pricing, and compensation. Initiatives sponsored by public entities or that are volunteer-driven tend to offer devices at no acquisition cost. This creates an imbalance in comparison to social enterprises, with employees preparing refurbished devices professionally that need to cover their professional costs. One way out is to reach agreements with these initiatives to segment intermediary entities (NGOs) that can pay for those that cannot really pay (for example, analysing their income-generating activities from annual activity reports). Another way is (when the target audience is the same) to raise funds to complement voluntary work with paid-for refurbished devices prepared by social enterprises. Moreover, when the general population are the beneficiaries, the pricing strategy for devices prepared for reuse by social enterprises coming from donations does not have to undercut (dumping) the existing secondhand-sector market prices. This allows the generation of an economic margin and more jobs.

Supporting results:

In our initiatives, we have found that the average sale price per refurbished device prepared by social enterprises is around $€ 40-140$ (depending on use value and refurbishment effort involved) for vulnerable people. As said before, the cost is either supported by the end user or a third party. eReuse has device-distribution channels for users, such as circula.cat and donalo.org, which coinciding with computer-donation campaigns by volunteer organisations) have noticed a significant drop in sales. This is the result of entities interested in acquiring these devices placing orders on one of these channels and then cancelling when they were able to acquire computers at no cost.

Our algorithm estimates price results in excessive imbalance between devices' use of value and what is offered by secondhandmarket prices of other commercial companies already operating in the market. For instance, we sold a Toshiba Tecra R950 laptop (i5, 4 GB RAM) for $€ 135$, while on a secondhand portal (Back Market) it was priced at $€ 412$. In contrast, our price for a Core 2 Duo, around $€ 40$, is closer to that of the secondhand market. This makes it difficult to operate, given these pieces are highly coveted.

Public procurement of circular devices or policies favouring such among private organisations and individuals is not systematised enough. The public sector should have a better understanding to take a role not only in donating, but more importantly in being part of the demand for the computers once refurbished. For instance, after a 2-year project trying to provide secondhand computers to public schools, even though it was technically feasible, the immense administrative and bureaucratic hurdles involved (contracting barriers) made it almost impossible. To achieve this type of goal, it is necessary to evolve a proposal together with the Department of
Digital Policies and Public Administration to assess the possibility of fitting its needs with public supply channels.

This is precisely one of the main conclusions of an analysis of business models in the reuse sector in Catalonia prepared by the Waste Agency of Catalonia within the framework of the European project Subtract (Interreg): "With the aim of increasing the economic viability of reuse centers, and reducing dependence on public subsidies, it is necessary to encourage financing through the sale of products and the provision of services. The administration is a consumer of many services that can be developed by reuse centers, and the increase in services provided by the administration is one of the demands made by the sector".

Supporting results:

Analysis of the pool of 1,079 computers under common management shows that $24 \%$ were acquired under the umbrella of public purchase of reused devices (devices paid for by social services, schools, or other public institutions), including two experiences under a servitised model, compared to $76 \%$ acquired by the private sector. Another result that supports this conclusion is that despite computer classrooms with secondhand devices running around the world, many barriers have been detected to making this possible in Catalonia. As a result of a 2-year project funded by the Catalan Waste Agency, few schools (five) adopted a semi-servitised model at a fair and sustainable price. This was due a number of factors, among which was the acquisition of computers in a cyclical way and under the umbrella of the Catalan government; therefore, it is necessary to have a system compatible with the public purchase system, centralised in the government's education department. It is necessary to adapt/align eReuse solutions with the public network (e.g., WiFi communication protocols, configuration of switches, prioritisation of services in communications, configuration of filtering, and security probes of the corporate network), The governmental digital policy did not provide maintenance for the reused devices, since the contracting was not done directly with this department, which generated problems about who was going to maintain the computers.

\subsection{Do circular devices really replace purchases of new devices?}

Demand substitution is an important concept in the circular economy. It happens only when a secondhand device satisfies demands and thus perfectly replaces a new device. In other words, it provides the same utility to the beneficiary, reducing the demand to manufacture more new devices. It has been claimed [27] that promoting reuse versus recycling (and consequently the need for manufacturing a new device from raw materials) may reduce environmental costs by $€ 45.20$ per PC. "Those social benefits are mainly generated in the re-use preparation process and distribution activities, whereas the re-use scenario displays a worse performance in energy consumption. The difference in the distribution stage during the second life cycle originates from the fact that the ready to reuse product is produced locally, while the brand-new product is manufactured and distributed from abroad, mainly Asia." Other research [3] has shown that devices with computational power "similar to that of first-hand consumption" result in an extension of 2-3 years of life with reuse, with an environmental impact of $39 \%-50 \%$ that of the purchase of a new device. 
In relation to this concept of substitutability, it is worth mentioning that the success factors in electronic reuse are related, among others, to the durability of the device, the limits of absolute obsolescence (that is, by the so-called designed lifetime), and the ability of a product to remain functional when faced with the challenges of normal service-life operation [6]. They are also conditioned by the so-called actual lifetime: the time from the moment a product is sold until it is discarded or replaced [41]. This term is conditioned, above all, by user behaviour: in how they use, maintain, and repair the device, in cultural and social influences, and in the norms and values they experience [8].

This broader approach is what is called product longevity. This influences the user, leading in some cases to products being discarded as a consequence of relative obsolescence or for preventable or avoidable causes before the product itself reaches its designed life or absolute obsolescence [4]. In fact, the so-called warm distribution, through intermediaries/NGOs who are in contact with the target audience and their digital needs, is a key factor in other schemes, such as that of the Amsterdam City Council [26], which refurbished 3,500 laptops to address the lack of infrastructure for vulnerable populations during COVID- through an ecosystem composed of 68 organisations.

In order to highlight the importance of the concept of longevity and final support to the end user to achieve it, we rely on the fact that the implementation of ICT in a territory is based on three fundamental pillars: infrastructure, training in use, and proximity of technology and services associated with it. These three pillars are complementary, and the poor implementation of any of them means not being able to take advantage of the possibilities offered by digital technology [2].

Supporting results:

All these considerations are relevant if we consider that some computers with low use value were discarded by end users. We have identified several main causes: mismatch between real usage needs and initial plans, expectation for Windows or certain applications (MS Office) while receiving computers with Linux operating systems, computers overloaded when reinstalled with Windows (Windows 10 in our recent cases), and lack of substantive competence in using Linux.

These mismatches mainly caused frustration, confusion, and "micro-anxiety" [44] crises among users blocking them to get work done. This article has other similar human-centred observations. Martin Heidegger wrote about "present-at-hand" tools versus "readyat-hand" tools- "The ready-at-hand tool works smoothly and supports the user's project - the user is practically unaware of the tool. The present-at-hand tool fails in some way, causing the user to shift attention away from their project"-to examine tools for a workaround or substitution to resolve the breakdown.

Dismissal due to avoidable or preventable causes, which also cause frustration, could be a rebound effect of our model preventing future purchases of reused computers. This is critical, as no social impact results in no environmental impact and no sustainable economic impact.

The presence of training in digitisation, from a critical perspective and based on our experience, is essential for technological appropriation, an important concept that helps to prevent the discarding of devices due to preventable causes. We think this concept is connected to impact generation while preventing rebound effects.

In our experience in Barcelona, 84 intermediary entities (NGOs, schools, and public institutions) have interceded between reuse centres and the final beneficiary. Many intermediary entities, especially NGOs and social and solidarity entities, have understood the model and appropriated it. This is the case for Sant Joan de Déu-Lleida, which since 2018 has acquired 53 devices to equip training rooms and apartments for young migrants over 18 years of age to help them in career opportunities in employment, such as mechanics, carpentry, and agriculture. Another example is the Punt de Referència NGO, with a similar objective, which purchased 28 devices. In both cases, the aim was enabling final beneficiaries with access to digital tools.

On the other hand, other entities did not find the model met their needs, especially with devices with lower usage value. As an example, an entity acquired 24 items (18 towers and six computer monitors). In a first purchase, they acquired six i3 towers (medium usage value) and six displays for $€ 697$, and in a second purchase 12 towers of low usage value for $€ 435$. When asked about this 2 years later, it reported that the towers from the second acquisition had been recycled "because they did not support the operating system" (it tried to replace the light Linux installed with Windows). This entity did not make any further acquisitions in the following years.

We have reported that several successful reuse/social experiences are strongly associated with final-user support, such as the acquisition by the Social Services of Barcelona of 22 devices to equip a school extra assistance room during the partial easing of COVID-19 restrictions (with final-user support from the Pare Manel Foundation) and the public purchase of 34 pieces of equipment for a school in Can Maiol, which then had the final-user support of Lleialtat Santsenca. An interesting case is that of the contracting by the District of Les Corts of refurbishing of their decommissioned equipment to Solidança for intern reuse in a neighbourhood facility.

Servitisation. Users might prefer a holistic computing solution offering a number of computing seats, instead of the ownership of certain devices [32]. This is the case in public computing facilities and computing classrooms for schools equipped with a number of computer devices and Internet connections with agreed characteristics maintained by a third party under a service-level agreement. We have done a pilot, which has initially looked promising, but the model is not yet mature and needs to be replicated, including analysis of the ease of enabling shared-computer classrooms with Internet-enabled devices (in a post-COVID-19 scenario), in order to rationalise and share resources.

Supporting results:

In 2018, donalo.org and Solidanca.cat developed a pilot project of servitisation with Barcelona Activa, an institution that opted for responsible public procurement of secondhand devices to equip two public facilities in two vulnerable neighbourhoods in Barcelona (Torre Baró and La Bordeta). The service is associated with the setup and maintenance of infrastructure and rental, not acquisition, of equipment. Despite the initial reluctance to use Linux or administrative and legal difficulties, the pilot is still working, even or devices of low value. Maintenance and support are key to extending the useful life of the devices. This is an interesting scenario, 
has many roles in the ecosystem to ensure maximum lifetime, and also has the highest benefit:cost ratio, according to the cost-benefit analysis carried out for all-use cases.

Professionalisation and cooperation across social actors (ecosystems) help to improve the ability to supply the demand when it appears. This was the case during the peak demand during the initial months of the COVID-19 pandemic.

Supporting results:

On 15 May 2020, when the Spanish government declared the first state of emergency, $15 \%$ of households in Catalonia did not have any type of computer at home (tablets, laptops or desktops) [39]. The old and unresolved issue of digital inequality was brought to the fore. Since the outbreak of the pandemic, the role of the state as guarantor of universal education (SGD4) has captured the public's attention when digital inequality became blatant educational inequality. The UNDP [11] warned that the rate of truancy rose to 1985 levels.

In order to alleviate the need for infrastructure for around 300,000 families with children in homeschooling and following the needs assessment for certain academic years carried out together with schools directors, the Generalitat of Catalunya, resorted to the old and well-known linear economy approach. According to [12], on 28 August a service contract valued at $€ 82$ million (before VAT) was awarded to two big companies due to the urgent need to provide 224,225 laptops and associated services. Furthermore, there are 30,000 additional devices to be ordered by the Spanish Ministry of Education and Red.es, as well as funds to be transferred to statesubsidised schools to purchase an additional 30,000 devices. In any case, the acquisition was insufficient to cover the 300,000 devices announced or for the needs of the rest of the population affected by digital inequality (the elderly, people in assisted living facilities, vocational students, migrants).

However, the solution that seemed easy a priori was not. On 18 January 2021, 8 months after the declaration of a state of emergency, only $5 \%$ of computers awarded had arrived. The Generalitat of Catalunya alleged there was a problem of lack of devices and components to manufacture them in the international market and that that was why the companies had not complied with the delivery terms established in the contracts [13]. This evidenced a lack of resilience in the linear economy-led civil society to take action. Several initiatives driven by civil society or companies and institutions were organised in Spain to alleviate the lack of access to computer devices (COVID Warriors, Labdoo, Madrid Futuro). Likewise, multiple initiatives were launched by companies in the name of corporate responsibility. For most, there was hardly any scientific literature on their social and environmental impact.

Perhaps, it would have been possible to meet unmet demand with financial public support for the 1,083 tower PC (mostly Core2Duo) that the circuit in Barcelona had at that time. It was an insufficient number to reach the 300,000 laptops/towers officially needed, but it is a decentralised and local option solution that can provide, not only local needs infrastructure, but also support reuse centres which, like many others, saw their already uncertain economic situation worsen during COVID-19.

During the months following home confinement, except July and August, the semi-relaxation of the state of emergency in Spain, eReuse software and services recorded a significant increase in devices traced and subsequently recirculated, with 544 under a common licence by the Barcelona circuit used in this COVID-19 context, as shown in Figure 2.

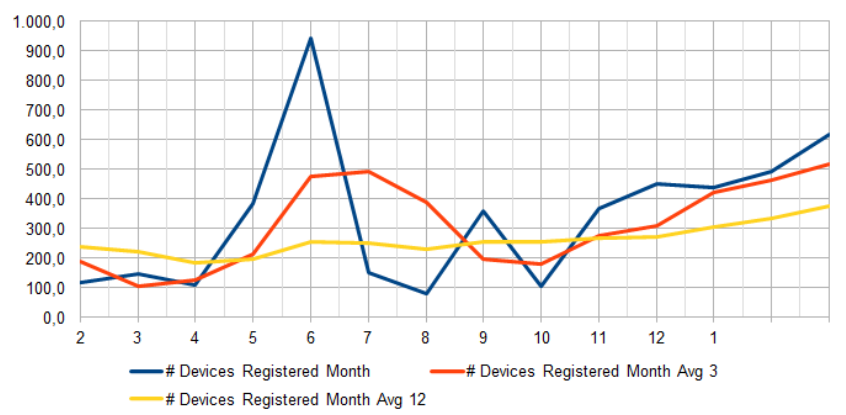

Figure 2: Evolution of devices registered per month in 2020.

At least 210 final beneficiaries received a device, the cost of which was paid for by third parties (UPC Centre for Development Cooperation and Catalan Waste Agency). The other 334 devices went to six schools and centres for direct distribution to families or facilities. The average price of a tower and monitor was $€ 40$ (not including WiFi-adapter costs and VAT), much lower than the price paid from the public purse, from $€ 285-385$ per device and associated services, depending on the operating system/device (Linkat, Chromebook, Windows 10, or dual system).

As mentioned in section 5.1, the Catalonian Waste Agency followed Barcelona City Council in taking advantage of the ecosystem and umbrella of the Barcelona circuit in order to distribute its decommissioned computers fairly among the entities of the territory. In this case, Andròmines andromines.net received the donation, and in the midst of the first wave of the Spanish pandemic managed to process 169 computers and reuse $89.3 \%$ of this among 31 families (1,425 minors and 1,465 young people as indirect beneficiaries). According to the Andròmines impact report, 24,336 kilos of $\mathrm{CO} 2$ could be generated if disposables were reused effectively.

For all these related operations, and due to the chaotic situation caused by COVID-19, cooperation among entities was key. To resolve the avalanche of requests, it required a great deal of logistic coordination among six reuse centres in Catalonia, at least 19 intermediates (NGOs, municipalities, schools, and public libraries), the contribution of a recycling company (a large cooperative [Abacus]), logistical and transport support (Banc dels Aliments), finalbeneficiary support for some cases (Lleieltat Santenca, Can Mayol, Pare Manel foundation, and Roquetes library) and maintenance (for two servitised pilots in Torre Baró and La Bordeta).

\subsection{What is the ideal balance between reuse and recycling?}

Decommissioned devices can either be prepared for reuse or should be recycled. Both have different costs and implications, and choices are key in raising an operational economic margin to optimise the cost:benefit ratio. Reaching a threshold in the volume of operations is also critical for economic feasibility. Although a computer may have a potential for reuse, it is not always possible to find a suitable audience. 
Too many devices will kill you! Refurbished devices with low use value in the warehouse without demand represent a sunk cost from preparation and a storage cost with no income. From this experience, we have come up with several solutions. Bulk offers for stock clearance (for instance, computers without an operating system installed priced at €20) are preferable to having batches of refurbished computers gathering dust and taking space in distribution warehouses. Look for other destinations: students can transfer them to another organisation experiencing demand (inter-cooperation). In cases of free donation to entities in the global south, keep in mind the suppliers and local reuse initiatives in order to avoid dumping It is always preferable to source from local initiatives. Recycling when nothing else can be done to save storage cost or the loss of value and usefulness over time is desirable. Initial storage and delayed triage for preparation or recycling should be addressed until there is demand.

Supporting results:

Thanks to our technological tools and the fact that reuse centres capture hours of use during the first life of the device, we have quantitatively determined intensity of use and differentiated between public and private donors. Devices donated by public administrations and distributed had an average of 17,049 use hours during their first life compared to an average of 12,770 use hours for devices donated by private companies. In addition, an analysis of processors, RAM, and hard drives showed that the devices donated by public administrations had low usage value compared to devices donated by the private sector, which had medium and high usage values. This gives an idea of the degree of efficient usage of electronic devices in public institutions, as we mentioned in section 5.1.

Devices with medium to high usage value are in higher demand that sometimes cannot be satisfied, which is not the case for low usage-value devices. At the implementation of eReuse, several reuse centres affiliated (federated) with eReuse in Barcelona suffered space-storage problems due to the accumulation of refurbished devices, with associated economic sustainability issues.

For this reason, services for the collection, transport, recovery, and destruction of waste electrical and electronic equipment according to the guidelines of Spanish Royal Decree 110/2015, to be compensated or by recycling companies for waste electrical and electronic equipment, should be considered. For instance, reuse centres in Catalonia are paid $€ 180$ per Ton for complete computers, $€ 800$ per Ton for a new-generation motherboard and €250 per Ton for hard drives.

Reliance on device donations alone for economic sustainability is hardly feasible, and does not allow a balancing of supply and demand. There is a need to combine donations (public or private) with other sources of devices to be able to plan investments or hiring of personnel. We have seen similar initiatives in Europe [40] that sometimes rely on the purchase of decommissioned laptops to meet demand, particularly during the pandemic.

Supporting results:

There was an initial tendency in eReuse circuits to accept devices without having assessed concrete demand (in particular, devices from public institutions, with more use hours and lower use value). The consequence has been that the pool of devices is not balanced, with too many computers with low demand and too few computers with high demand to meet supply, especially those with processors below the i3 level or desktops. We were asked for laptops and computers with high usage value, but in many cases we only had Core 2 Duo desktops!

In donations of low use-value computers, it is important to value additional services, such as data-erasure services. It is important to know how to say no when these conditions are not met. Supporting results:

Lack of awareness has been detected on the part of donors and lack of clear distinction between what it means to generate positive externalities (jobs and computers at fair prices with the donation of devices of medium/high use value) and what it means to generate negative externalities (transportation, storage, and data wiping, without retribution or participation in the demand for donations of low computational-power material).

\subsection{Scale, replication}

When moving from theory to practice, and even more to sustainable practice, scale and capacity for replication is key. Circuits work as long as there are the minimum stakeholders (donors, refurbishment technicians, and users) with a minimally stable demand and supply to ensure efficient processing (ideally at industrial scale). The process must be economically, socially, and environmentally sustainable to persist and satisfy all parts' key roles, for example maintenance and support for final users are needed to overcome the barriers related to user behaviour and to ensure longevity and substitutability of primary materials.

We are involved in different processes of trial and replication of the model in other regions. There are local initiatives in Argentina, Spain, and several African countries that are in different stages of this development process. Based on the replication experience in Madrid, we can conclude that it is difficult to reach a feasibility threshold with a restricted target audience. To become a viable option in the market, always under the principles of eReuse, adaptability adaptation to environmental conditions is a requirement [43] that requires frequent and agile experimentation and self-evaluation, testing and verifying alternative models and failing and changing quickly. In this sense, we try to receive experimental feedback to adapt the software to needs and practices, not the other way around.

The level of funding required for economic sustainability of refurbishment work and the sale of reused computers is a limiting factor in replicating the process in other regions. This requires a critical set of key complementary roles. There is a need for initial training, development, and certification of good practices, coordination of the tasks and management of a stable device demand, and supply at prices adapted to the local economy. Software tools and services have to be adopted, translated, developed, and maintained locally to keep the scheme effective, affordable, and accountable.

The trial and replication process starts with one local organisation close to the issue that understands the global interest in the circular economy and sees a viable local need for reused devices, benefits for the local population as final beneficiaries, and refurbishment jobs. They can start from a once-off action to match the demand for computer devices with a single donation from a local public or private organisation or a campaign to collect computers from individual citizens. After a successful volunteer-driven 
effort to prepare and deliver these computers to final beneficiaries, a group of local people and organisations may see clearly the potential of bootstrapping a structured process to promote regular donations, refurbishment, recycling, and sale with local sponsors of these devices to beneficiaries.

Political commitment matters, as shown by the direct collaboration and involvement of the Barcelona City Council. As a result, it is important to align actions with public administrations as a necessary ingredient to define supportive policies, create subsidy programs, and involve them in supporting the sustainability of these projects. To avoid the broken-window fallacy, in which society loses the value of uselessly destroyed objects, cooperation among ecosystem actors is needed, as discussed in subsection 5.2, but sometimes this is complex and can be a burden.

The fact that all circuits operate under the umbrella of eReuse helps in the creation and sharing of tools, protocols, and best practices, as well as having a common voice for awareness raising. One of the most important challenges for the future is governance, which has multiple challenges, such as more inclusion, participation of other key stakeholders, better internal communication, consolidation of protocols, and other coordination mechanisms.

Supporting results:

There have been several cases of cooperation among federated entities in both circuits-Madrid and Barcelona. In Madrid, they refer donations to the Barcelona circuit, and vice versa, which increases supply. However, each circuit has its own peculiarities.

In Madrid, they do not use the formula of commodatum (shared pool, shared property) to formalise the sale of devices. This is why agreements with donors and beneficiaries involve a lot of bureaucracy. It is necessary to translate them into practical situations. They are useful at a conceptual level, but they have been found to be too theoretical and not very functional. In practice, they tend to be complex to implement: not understood by donors and final users, as shown by limited feedback or follow-up by users. In comparison, an economic deposit as a guarantee appears to be a good simplification, resulting in a minimum commitment on the part of the final user, who can get the money back if they are able to recycle the device correctly and report or return it.

Until recently and despite several attempts, there has not been political commitment from municipalities to donate computers or responsible public procurement. Nonetheless, in 2021 the Asociación Cultural La Kalle-Reutilitzak and Lyma, the municipal waste company of the Getafe City Council (Madrid region), signed an agreement to create and coordinate a circuit for the reuse of electronic devices that will be made available to the general public, NGOs, schools, and institutions at fair prices. This circuit will be fed by devices valid for reuse received at refuse points in the municipality and donations from companies and individuals.

The priority of the Madrid circuit, and especially of the Asociación Cultural La Kalle-Reutilitzak, is social, so of the 950 devices, 300 were donated free of charge to entities that proved, via annual activity reports, not to have income-generating activities. They take advantage of the reuse process to train vulnerable young people in the neighbourhood.

The Barcelona circuit supports end users, both in group and individual training, using such channels as Telegram and Jitsi. In this regard, it emphasises the reinforcement of free software to strengthen the digital competence of beneficiaries, in order to develop their skills in a critical way and achieve technological appropriation of the eReuse model. For that, it has set up a classroom in a school centre with 65 low value-use devices that (thanks to maintenance and incident support) are still in operation. However, this is an isolated case, since the schools work with other companies (on an hourly basis), are not interested in annual contracts, and if contracts do occur, the budgets are very low.

The Madrid circuit is an example of how the circular economy is capable of creating resilient strategies with multiplier effects if articulated under a participatory and transparent logic among various spheres. We understand multiplier effects in this context as the capacity of the different economic and social actors involved in ecosystems based on the circular economy of electronic devices to educate in terms of values and to explain to citizens the structural causes of inequality, the environmental crisis, and the consequences of technological training in the development of creativity and digital skills. Digital skills and digital understanding are two other aspects of the multidimensional nature of the digital divide that often go unnoticed in the limited budgets of public administrations, with myriad barriers that, in cases like Madrid, are solved by social entities.

\subsection{From theory to practice}

When we look back to our initial work [24] and the Limits paper [21], we do not find major differences. Our previous work built on previous practice, the service-learning experience of artisanal preparation of computers for reuse. One difference is that the writing a shared-property agreement (commodatum) can generate fear and costs for both the reseller and the buyer. In the B2B case (from reseller to business), the signatory is afraid to do so because they sign on behalf of their organisation, and if anything happens, they are afraid to appear as the person in charge. To solve this, in public procurement contracts, this is revised by lawyers, but this becomes an extra cost for both parties, who may not have the time to study these agreements.

To encourage the recipient to return the equipment for reuse or recycling, it appears to be better to introduce an economic incentive (e.g. a deposit, something that works well in other sectors) as a conditional reward, to be returned when the device is returned and reported. However, this should be tested and confirmed. To encourage a reseller to report the traceability and impact of equipment, we are creating impact certificates to help refurbishment technicians differentiate cases.

\section{RELATED WORK}

Other authors have published work on barriers to electronic reuse. For example, [5] points to some barriers that we have also developed in this article related to consumer attitudes and perceptions about reliability and product lifetimes, which translate into inadequate demand for secondhand goods. They also discuss other important points regarding excess supply and lack of demand: "many people are willing to donate (for reuse) but are reluctant to purchase." This work also notes that the interconnected relationship of this barrier and two others (producer reluctance and unsuitable collection infrastructure) influences the quality and quantity of items available for reuse and impact on the market for used goods. For that, 
a holistic approach is required, seeking to limit any unintended consequences at a different point in a product's life.

With regard to the latter issue, other authors have argued that circular economy activities can increase overall production, which can partially or fully offset their benefits [50]. Some of the mechanisms that cause circular economy rebound are the limited ability of secondary products to substitute for primary products and price effects. regarding the first mechanism, [7] cautioned that convincing consumers to extend product lifespan can be difficult, due to stigma about owning outdated products and that consumers may lack the care and attention required to maintain and repair products. To avoid circular economy rebound, the authors warned that "strategies that market secondary goods as specialised products sold either to niche markets or at vastly different price points are likely to be ineffective at creating meaningful environmental benefit. Instead, companies must market secondary goods in the same way as primary goods-using similar channels, touting similar benefits, and reaching similar customers".

Other interesting considerations about rebound effects are those pointed out by [33], who concludes that gains arising from robust repair networks are unlikely on their own to overcome the rebound effects related to aggregated demand for energy. Instead, the author points to fundamental changes in design, manufacturing, and business models before devices are in the hands of consumers. The world in 2030, in which repair could be a key process, would be based not on efficiency, but sufficiency. The importance of rebound effects in the development of new digital services and technologies is also argued by [10], who warns that the skill-rebound effect is very typical for digital technologies, so this needs to be better understood.

\section{CONCLUSIONS}

eReuse has built a model of reuse circuits that works in different cities and regions in Spain. The model appears to be effective in being economically, socially, and environmentally sustainable. Coordination among complementary stakeholders helps ensure the complete set of capabilities and skills to bootstrap a local circular economy with digital devices. The software allows improvement in efficiency (processing time) and quality of the refurbishment. Collected data allow the calculation impacts and reporting of these to donors and the public in general. Open datasets are useful for activists and governments in encouraging manufacturers and device owners to act responsibly for the challenge of developing a circular economy of digital devices that make ICT part of the solution to sustainable development (less inequality, less environmental impact), and not part of the problem (from the environmental impact of premature recycling devices after first use and lack of impact assessment).

From a critique about previous eReuse papers, and based on our accumulated experience in the last few years reusing computers from public administrations and companies, we have identified some other key points that can help in providing computing services that really substitute the demand at affordable prices for excluded and not excluded people and that are necessary to create social, economic, and environmental impact.

In economic terms, we need to break the poverty-reuse paradigm for the circular economy to be feasible at scale to open to the general market without dumping the secondhand sector, while at the same time ensuring through sponsors access of vulnerable people to a pool of devices shared as a commons, and consider an operating margin for optimal cost:benefit- ratio: we need to ask ourselves who is going to pay the circular cost before accepting any donation, balance supply with other sources, not only donations, charge for data-wipe services if devices have low usage value, and recycle when nothing can be done with the device before it stays in a warehouse for months. As a result, recycling is economically positive if there is a good deal, and storage is a continued loss.

Regarding social and environmental factors, key factors like maintenance and support for final users are needed to overcome barriers related to relative obsolescence. Retaining the maximumpossible use value of an electronic device through reuse is conditioned not only by the durability of the product but also by its longevity, in final-user behaviour, and norms and values. Taking into account these new scopes and limitations, it is important to avoid creating a rebound effect on people already penalised by inequality and to offer circular solutions that really replace new products.

Replicating the model in Madrid has given us experience and lessons learnt on how to adapt tools to the conditions of each community, the need to simplify and automate to avoid time-consuming protocols, the importance of having public support, and the importance of working with public and private entities to raise awareness, as well as communications and reporting. The model can be replicated in other locations with similar contexts or under more controlled conditions in localities with a lower development index.

There is future work to be done on understanding the concept of durability versus longevity and finding mechanisms of analysis and experience to validate this. However, we still need to find out to what extent our model reduces the purchasing of new devices. Also, there is need for more analysis of the boundary between reuse and recycling, costs versus benefits, and practical policies related to digital inclusion and the effectiveness of a circular economy of digital devices. Finally, more research on environmental impact is needed, as well as determining clear differences between the financial and environmental costs of recycling and reuse in our model. In this sense, it is also necessary to evaluate the rebound effects related to reuse to be sure that the model is capable of contributing to regenerating natural systems.

\section{ACKNOWLEDGMENTS}

This work was partially funded by the Spanish government under contracts PID2019-106774RB-C21, and PCI2019-111851-2 (LeadingEdge Chist-era), the NGI-DLT4EU, NGI-Policy, NGI-Atlantic projects, Subtract (Interreg Europe), and the Waste Agency of the Catalonia.

Many organisations and people have participated in the eReuse circuit and have contributed to improving the model: Pangea.org for supporting the eReuse project since its initial idea, the Centre of Cooperation for Development UPC, the City Councils of Barcelona, Sant Boi and Getafe, and the entities part of eReuse: Asociación Cultural La Kalle-Reutilizak, Solidança, Donalo.org, AndròminesAlencop, Fundación Esplai, Trinijove, TXT-UPC, Banc dels Aliments, Abacus, Sant Joan de Déu-Terres de l'Ebre, Punt de referència, among others. 


\section{REFERENCES}

[1] Thomas Adisorn, Lena Tholen, and Thomas Götz. 2021. Towards a digital product passport fit for contributing to a circular economy. Energies 14, 8 (2021), 2289. https://doi.org/10.3390/en14082289

[2] Daniel Orlando Icaza Álvarez, Gerardo Eugenio Campoverde Jiménez, Pablo D Arias, and Diego Verdugo. 2019. El analfabetismo tecnológico o digital. Polo del Conocimiento: Revista científico-profesional 4, 2 (2019), 393-406.

[3] Hampus André, Maria Ljunggren Söderman, and Anders Nordelöf. 2019. Resource and environmental impacts of using second-hand laptop computers: A case study of commercial reuse. Waste Management 88 (2019), 268-279. https: //doi.org/10.1016/j.wasman.2019.03.050

[4] John Bachér, Yoko Dams, Tom Duhoux, Yang Deng, Tuuli Teittinen, and Lars Fogh Mortensen. 2020. Electronic products and obsolescence in a circular economy. Eionet. Retrieved Abril 1, 2021 from https://www.eionet.europa.eu/etcs/etcwmge/products/electronics-and-obsolescence-in-a-circular-economy Eionet Report - ETC/WMGE 2020/3

[5] Christine Cole, Alex Gnanapragasam, Tim Cooper, and Jagdeep Singh. 2019 Assessing barriers to reuse of electrical and electronic equipment, a UK perspective. Resources, Conservation \& Recycling: X 1 (2019), $100004 . \quad$ https: //doi.org/10.1016/j.rcrx.2019.100004

[6] Tim Cooper. 1994. Beyond recycling, the longer life option. NTU. Retrieved Abril 1, 2021 from http://irep.ntu.ac.uk/id/eprint/24666/1/201125_7266\%20Cooper\% 20Publisher.pdf New Economics Foundation.

[7] Tim Cooper. 2005. Slower consumption reflections on product life spans and the "throwaway society". Journal of industrial Ecology 9, 1-2 (2005), 51-67. https://doi.org/10.1162/1088198054084671

[8] Tim Cooper. 2016. Longer lasting products. Alternatives to the throwaway society. (1st ed.). Routkedge, London, UK. https://doi.org/10.4324/9781315592930

[9] Copper8. 2019. Circular Revenue Models: Required Policy Changes for the Transition to a Circular Economy. Copper8. Retrieved Abril 1, 2021 from https//copper8.com

[10] Vlad C. Coroamă and Daniel Pargman. 2020. Skill Rebound: On an Unintended Effect of Digitalization. In Proceedings of the 7th International Conference on ICT for Sustainability (Bristol, United Kingdom) (ICT4S2020). Association for Computing Machinery, New York, NY, USA, 213-219. https://doi.org/10.1145/ 3401335.3401362

[11] Programa de Desarrollo Sostenible de las Naciones Unidas. 2020. COVID-19 and Human Development: Assessing the Crisis, Envisioning the Recovery. UNDP. Retrieved Abril 1, 2021 from http://hdr.undp.org/en/hdp-covid

[12] Departament d'Educació. Generalitat de Catalunya. 2020. COVID19 and Human Development: Assessing the Crisis, Envisioning the Recovery. Generalitat de Catalunya. Retrieved Abril 1, 2021 from https://contractaciopublica.gencat.cat/ecofin_pscp/AppJava/awardnotice. pscp?reqCode $=$ viewPcan\&idDoc $=67900379$ \&lawType $=3$

[13] Soledad Dominguez. 2021. El departament d'educació només ha lliurat un 5\% dels 300000 ordinadors compromesos per als alumnes. Cadena Ser. Retrieved Abril 1, $2021 \mathrm{from} \mathrm{https://cadenaser.com/emisora/2021/01/19/sercat/1611077760} 555340$. html Cadena Ser.

[14] Interreg Europe. 2021. Socially minded platform for the reuse and recycling of Electrical and Electronic Equipment (EEE). Interreg Europe. Retrieved Abril 1, 2021 from https:/www.interregeurope.eu/policylearning/news/11475/sociallyminded-platform-for-the-reuse-and-recycling-of-electrical-and-electronicequipment-eee/ Policy Learning Platform.

[15] Johann Fellner, Jakob Lederer, Christoph Scharff, and David Laner. 2017. Present potentials and limitations of a circular economy with respect to primary raw material demand. Fournal of Industrial Ecology 21, 3 (2017), 494-496. https: //doi.org/10.1126/science.162.3859.1243

[16] Vanessa Forti, Cornelis Peter Baldé, Ruediger Kuehr, and Garam Bel. 2020. The Global E-waste Monitor 2020: Quantities, flows and the circular economy potential. United Nations University (UNU)/United.

[17] Marcus Foth. 2006. Network action research. Action Research 4, 2 (2006), 205-226.

[18] Amazon Aid Foundation. 2011. Rio de oro. Amazon Aid. Retrieved Abril 1, 2021 from https://amazonaid.org/rio-de-oro/

[19] Ellen McArthur Foundation. 2017. What is the circular economy? Ellen McArthur Foundation. Retrieved Abril 1, 2021 from https://www.ellenmacarthurfoundation. org/circular-economy/what-is-the-circular-economy

[20] David Franquesa and Leandro Navarro. 2017. Sustainability and participation in the digital commons. ACM Interactions 24, 3 (2017), 66-69. https://doi.org/10. $1145 / 3058139$

[21] David Franquesa and Leandro Navarro. 2018. Devices as a Commons: Limits to Premature Recycling. In Proceedings of the 2018 Workshop on Computing within Limits (Toronto, Ontario, Canada) (LIMITS '18). Association for Computing Machinery, New York, NY, USA, Article 8, 10 pages. https://doi.org/10.1145/3232617. 3232624

[22] David Franquesa and Leandro Navarro. 2020. eReuse datasets, 2013-10-08 to 201906-03. UPC. Retrieved Abril 1, 2021 from https://dsg.ac.upc.edu/ereuse-dataset

[23] David Franquesa, Leandro Navarro, Stephan Fortelny, Mireia Roura, and Jordi Nadeu. 2019. Circular consumption and production of electronic devices: an approach to measuring durability, upgradeability, reusability, obsolescence and premature recycling. In The European Roundtable for Sustainable Consumption and Production - Circular Europe for Sustainability, Barcelona. UPC, Barcelona, Spain, 196-197.

[24] David Franquesa, Leandro Navarro, David López, Xavier Bustamante, and Santiago Lamora. 2015. Breaking Barriers on Reuse of Digital Devices Ensuring Final Recycling. In 29th International Conference on Environmental Informatics, EnviroInfo 2015 / 3rd International Conference on Information and Communication Technology for Sustainability, ICT4S 2015, Copenhagen, Denmark. Atlantis Press, Amsterdam, Netherlands, 281-288.

[25] Anastasius Gavras, Arto Karila, Serge Fdida, Martin May, and Martin Potts. 2007. Future internet research and experimentation: the FIRE initiative. Comput. Commun. Rev. 37, 3 (2007), 89-92. https://doi.org/10.1145/1273445.1273460

[26] Nicole Goedhart, Paulien Tensen, and Christine Dedding. 2020. Tedereen verbonden' met een refurbished laptop. Amsterdam City Council. Retrieved Abril 1, 2021 from https://openresearch.amsterdam/nl/page/66162/evaluatie-iedereenverbonden

[27] Xose Manuel González, Miguel Rodríguez, and Yolanda Pena-Boquete. 2017. The social benefits of WEEE re-use schemes. A cost benefit analysis for PCs in Spain. Waste Management 64 (2017), 202-213. https://doi.org/10.1016/j.wasman.2017. 03.009

[28] Nick Hanley. 2001. Cost-benefit analysis and environmental policymaking. Environment and Planning C: Government and Policy 19, 1 (2001), 103-118. https: //doi.org/10.1068/c3s

[29] Garrett Hardin. 1968. The Tragedy of the Commons. Science 162, 3859 (1968), 1243-1248. https://doi.org/10.1126/science.162.3859.1243

[30] ITU-T. 2016. Circular economy in information and communication technology; definition of approaches, concepts and metrics. ITU-T. Retrieved Abril 1, 2021 from http://handle.itu.int/11.1002/1000/13151 Supplement 28 to ITU-T L-series Recommendations.

[31] ITU-T. 2020. NGHG emissions trajectories for the ICT sector compatible with the UNFCCC Paris Agreement,. ITU-T. Retrieved Abril 1, 2021 from https: //www.itu.int/rec/T-REC-L.1470-202001-P Recommendation ITU-T L.1470.

[32] ITU-T. 2021. The potential impact of selling services instead of equipment on waste creation and the environment - Effects on global information and communication technology. ITU-T. Retrieved Abril 1, 2021 from https://www.itu.int/rec/T-RECL.1024 Recommendation ITU-T L.1024.

[33] Josh Lepawsky. 2020. Towards a World of Fixers Examining Barriers and Enablers of Widely Deployed Third-Party Repair for Computing within Limits. In Proceedings of the 7th International Conference on ICT for Sustainability (Bristol, United Kingdom) (ICT4S2020). Association for Computing Machinery, New York, NY, USA, 314-320. https://doi.org/10.1145/3401335.3401816

[34] V. Masson-Delmotte, P. Zhai, H.-O. Pörtner, D. Roberts, J. Skea, P.R. Shukla, A. Pirani, W. Moufouma-Okia, C. Péan, R. Pidcock, S. Connors, J.B.R. Matthews, Y. Chen, X. Zhou, M.I. Gomis, E. Lonnoy, T. Maycock, and M. Tignor andT. Waterfield (eds.). 2018. Special report Global warming of $1.5^{\circ} \mathrm{C}$. Technical Report. Intergovernmental Panel on Climate Change. https://www.ipcc.ch/sr15/

[35] Donella Meadows, Dennis Meadows, Jørgen Randers, and William Behrens. 1972. The Limits to Growth; A Report for the Club of Rome's Project on the Predicament of Mankind. Universe Books, Winterthur, Switzerland.

[36] Metabolic.nl. 2019. The Seven Pillars of the Circular Economy. Metabolic. Retrieved Abril 1, 2021 from https://www.metabolic.nl/news/the-seven-pillars-ofthe-circular-economy/

[37] United Nations. 1987. Our common future. United Nations. Retrieved Abril 1, 2021 from http://www.un-documents.net/our-common-future.pdf , also known as The Brundtland Report.

[38] Leandro Navarro. 2021. Policy in Practice Fund: Reducing the digital divide by improving the circular economy for devices. NGI PIP. Retrieved May 20, 2021 from https://research.ngi.eu/policy-in-practice-fund-reducing-the-digitaldivide-by-improving-the-circular-economy-for-devices/ PiP.

[39] National Statistics Institute of Spain. 2019. Encuesta sobre Equipamiento y Uso de Tecnologías de Información y Comunicación en los hogares 2019. National Statistics Institute of Spain. Retrieved Abril 1, 2021 from https://www.ine.es/up/jeFtuUrziA

[40] Michael J. Oghia. 2018. Digital Inclusion Luxembourg: A holistic approach to refugee inclusion, access, and sustainability. ITU-T. Retrieved Abril 1, 2021 from https://news.itu.int/digital-inclusion-luxembourg-a-holistic-solution-torefugee-inclusion-access-and-sustainability/ ITUNews Magazine.

[41] Masahiro Oguchi, Shinsuke Murakami, Tomohiro Tasaki, Ichiro Daigo, and Seiji Hashimoto. 2010. Lifespan of commodities, part II: Methodologies for estimating lifespan distribution of commodities. Fournal of Industrial Ecology 14, 4 (2010), 613-626. https://doi.org/10.1111/j.1530-9290.2010.00251.x

[42] Elinor Ostrom. 1990. Governing the commons: The evolution of institutions for collective action. Cambridge university press, Cambridge, UK.

[43] Elinor Ostrom. 2008. The challenge of common-pool resources. Environment: Science and Policy for Sustainable Development 50, 4 (2008), 8-21. https://doi.org/ 10.3200/ENVT.50.4.8-21

[44] Sharon Helmer Poggenpohl. 2020. Waste and Agency in the Digital Era: Who's in Charge? She fi: The Journal of Design, Economics, and Innovation 6, 3 (2020), 
331-344. https://doi.org/10.1016/j.sheji.2020.07.003

[45] Kate Raworth. 2012. A Safe and fust Space for Humanity: Can we live within the doughnut? Oxfam. Retrieved Abril 1, 2021 from https://policy-practice.oxfam.org/resources/a-safe-and-just-space-forhumanity-can-we-live-within-the-doughnut-210490/

[46] Johan Rockström, Will Steffen, Kevin Noone, Åsa Persson, F Stuart Chapin, Eric F Lambin, Timothy M Lenton, Marten Scheffer, Carl Folke, Hans Joachim Schellnhuber, et al. 2009. A safe operating space for humanity. Nature 461, 7263 (2009), 472-475
[47] Statista. 2021. Number of smartphones sold to end users worldwide from 2007 to 2021. Statista. Retrieved Abril 1, 2021 from https://www.statista.com/statistics/ 263437/global-smartphone-sales-to-end-users-since-2007/

[48] Jo A Tacchi, Don Slater, and Gregory Hearn. 2003. Ethnographic Action Research: A User's Handbook. UNESCO, New Delhi, India.

[49] Wikipedia. 2021. Sustainable development. Wikipedia. Retrieved Abril 1, 2021 from https://en.wikipedia.org/wiki/Sustainable_development

[50] Trevor Zink and Roland Geyer. 2017. Circular economy rebound. Fournal of Industrial Ecology 21, 3 (2017), 593-602. https://doi.org/10.1111/jiec.12545 